\title{
CANULACIÓN DE LA ARTERIA INNOMINADA PARA CONEXIÓN A CIRCULACIÓN EXTRACORPÓREA EN CIRUGÍA DE LA AORTA*
}

\author{
Drs. Enrique Seguel S. ${ }^{1,2}$, Aleck Stockins L. ${ }^{1,2}$, Roberto González L. ${ }^{1,2}$, \\ Andrés Jadue T. ${ }^{2}$, Emilio Alarcón C. ${ }^{1,2}$ \\ Departamento de Cirugía Facultad de Medicina Universidad de Concepción. \\ 2 Centro Cardiovascular Hospital Guillermo Grant Benavente. \\ Concepción, Chile.
}

\begin{abstract}
\section{Innominate artery cannulation for aortic artery surgery with} extracorporeal circulation
\end{abstract}

Surgery of the ascending aorta and its arc with extracorporeal circulation require an arterial cannulation that assures and adequate systemic circulation and reduces the risk of neurological complications. A peripheral cannulation such as the femoral artery, a central extra-thoracic approach such as axillary, subclavian or carotid arteries or a central intra-thoracic approach such as aorta and innominate arteries, are valid alternatives. Innominate artery cannulation can be carried out without the need of a second incision. It is an easily accessible and large bore vessel. It allows an anterograde brain and visceral flow. In cases of aortic arch surgery, the clamping of the artery in its origin, allows a selective anterograde brain perfusion. The surgical technique for innominate artery cannulation is herein described.

Key words: Aorta, innominate artery, cannulation.

\section{Resumen}

La cirugía de la aorta ascendente y cayado con circulación extra corpórea requiere de una canulación arterial que permita asegurar una perfusión sistémica adecuada y disminuir las complicaciones neurológicas. El sitio ideal de canulación es aún un tema de discusión entre los cirujanos. Entre las alternativas de abordaje están la canulación periférica (arteria femoral), la canulación central extratorácica (arterias axilar, subclavia o carótidas) y la canulación central intratorácica (aorta, arteria innominada). La canulación de la arteria innominada es una técnica que se puede realizar sin necesidad de una segunda incisión, es un vaso de fácil abordaje y habitualmente de gran calibre. Permite entregar un flujo cerebral y visceral anterógrado y, en caso de cirugía sobre el cayado, el pinzamiento de la arteria a nivel de su origen permite entregar una perfusión cerebral selectiva anterógrada. Se describen la técnica quirúrgica y los reparos operatorios que nos parecen importantes en su realización.

Palabras clave: Canulación, circulación extra corpórea, arteria innominada, cirugía aórtica, protección cerebral, perfusión cerebral selectiva anterógrada.

\footnotetext{
*Recibido el 19 de mayo de 2014 y aceptado para publicación el 22 de julio de 2014.

Los autores no refieren conflictos de interés.

Correspondencia: Dr. Enrique Seguel S. enseguel@udec.cl
} 


\section{Introducción}

La adecuada protección cerebral ha sido el gran desafío de la cirugía de la aorta torácica, especialmente cuando se debe trabajar sobre el arco aórtico.

Desde los inicios de esta cirugía se han utilizado diferentes sitios de canulación arterial para la conexión de los pacientes a circulación extra corpórea. Las diferentes técnicas pueden ser clasificadas como remotas (arteria femoral); centrales extratorácicas (arterias axilar, subclavia y carótidas) o centrales intra torácicas (aorta, arteria innominada). Todas estas técnicas tienen ventajas y desventajas y son utilizadas en diferentes proporciones por los diferentes centros para este tipo de intervenciones.

La técnica de canulación femoral se utiliza desde los años 50 y es probablemente la más extendida para la cirugía de la aorta. Entre sus ventajas están su fácil acceso y rapidez de instalación. Puede ser llevada a cabo antes o simultáneamente con la abertura del tórax. Puede ser realizada de manera directa (por disección) o por vía percutánea, y existen variados modelos de cánulas que permiten asegurar un flujo adecuado a todos los pacientes. Esta técnica no está exenta de complicaciones, entre las que destacan el daño vascular y la isquemia de la extremidad. Debido a que el flujo por la aorta es retrógrado, se puede producir ateroembolismo hacia los vasos cerebrales y existe un riesgo de malperfusión visceral. $\mathrm{Su}$ uso es limitado en pacientes con enfermedad arterial obstructiva de los territorios aorto-iliaco y femoral. El principal inconveniente es que no permite perfusión cerebral selectiva en caso de cirugía sobre el cayado, y cuando se utiliza esta técnica, la protección cerebral se limita a la hipotermia profunda durante un período de paro circulatorio que no puede exceder los $30 \mathrm{~min}$. Probablemente por esta causa, su uso en disección aórtica aguda tipo A se asocia a mayores tasas de morbilidad neurológica que las otras técnicas ${ }^{1-5}$.

La canulación directa de las arterias carótidas es una técnica de fácil acceso, rápida y que permite una excelente perfusión cerebral. Permite la manipulación del cayado sin necesidad de detener la circulación. Puede hacerse a ambos lados o en uno solo si el polígono de Willis es suficiente. Está indicada en pacientes con compromiso de los troncos supra aórticos en caso de disección aguda y en pacientes con anormalidades del arco (arteria lusoria). Cuando se usa en forma exclusiva, no permite la medición de la presión arterial por vía radial. Puede combinarse con otras técnicas, como la canulación femoral, para asegurar el flujo visceral ${ }^{6,7}$.

La canulación de las arterias axilar y subclavia es una técnica alternativa que se ha utilizado desde los años 90. Es una técnica relativamente simple y rápida que permite entregar un flujo anterógrado y la posibilidad de entregar perfusión cerebral selectiva en caso de paro circulatorio. No interfiere el campo operatorio. Existe el riego de complicaciones como la lesión vascular, del plexo braquial, o la isquemia de la extremidad. Debido a que se trata de vasos más frágiles y de menor calibre, es recomendable la interposición de una prótesis vascular que permite proteger la arteria y mantener la irrigación de la extremidad, aunque esto puede alargar un poco el procedimiento ${ }^{8-11}$.

La canulación directa de la arteria innominada fue descrita por Michael Banbury y Delos Cosgrove el año $2000^{12}$. Es una técnica que permite realizar la intervención en un solo campo operatorio. Puede hacerse antes de abrir el pericardio. Debido al tamaño de la arteria, permite la utilización de cánulas de diámetro grande. Entrega una perfusión anterógrada y permite la realización de perfusión cerebral selectiva en caso de un paro circulatorio. Entre sus desventajas está el que este vaso no puede utilizarse en caso de disección, aneurisma o calcificación del mismo $^{12-16}$.

Iniciamos el uso de esta técnica en nuestro equipo a fines de 2010 y el objetivo de esta comunicación es compartir los detalles técnicos que nos parecen más importantes.

\section{Técnica quirúrgica}

El estudio preoperatorio es el habitual de los pacientes de acuerdo a su patología y debe incluir un angioTC de tórax, pues permite diagnosticar la presencia de malformaciones o anormalidades congénitas (arteria lusoria; tronco bovino), aneurismas, enfermedad aterosclerótica y/o disección de la arteria innominada. Estas situaciones pueden contraindicar la técnica.

Los pacientes se intervienen bajo anestesia general. La monitorización es la estándar y depende de la patología, pero es importante que la línea de presión arterial debe ser instalada en la extremidad superior derecha.

Los pacientes se preparan de la forma habitual, incluyendo las dos extremidades inferiores en el campo con el fin de tener acceso a los vasos femorales en caso de necesidad de canulación de ellos.

El acceso a la arteria innominada se realiza mediante esternotomía media, la cual debe llegar a la horquilla esternal. Una vez instalado el retractor esternal se procede a la disección de la grasa mediastínica en la línea media hacia cefálico de la vena innominada (Figura 1). La arteria normalmente es de gran calibre $(1,5-2 \mathrm{~cm})$ y tiene una dirección oblicua que incluso puede se horizontal, especial- 


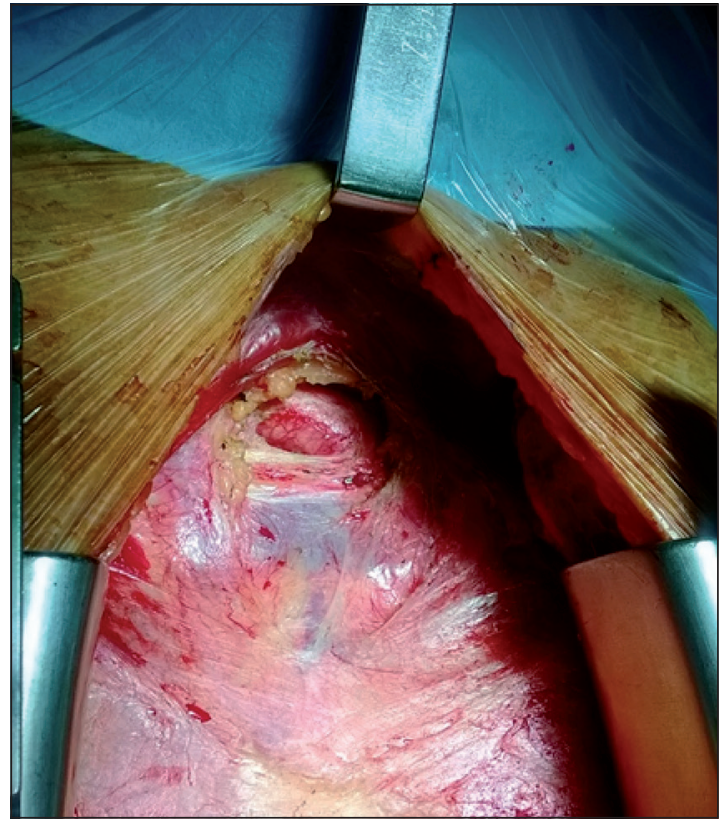

Figura 1. Disección del mediastino anterior y exposición de la arteria innominada.

mente cuando su origen se ha desplazado hacia la izquierda por la presencia de un aneurisma de la aorta ascendente.

Una vez expuesto el vaso se procede a la heparinización completa y a la confección de dos jaretas concéntricas con sutura de polipropileno 5-0 que se aseguran con torniquetes (Figura 2).

El procedimiento de canulación es similar al de la aorta: Se procede a seccionar la adventicia del vaso dentro de las jaretas, se realiza una incisión con bisturí hoja 11 y procede al implante de la cánula. Utilizamos para esta técnica una cánula recta biselada (DLP ${ }^{\circledR}$ Medtronic, Inc. Minneapolis, MN, USA) de 20 ó 22 French. El bisel de la cánula debe quedar apuntando hacia el cayado aórtico. Una vez completada la canulación se procede al cierre de los torniquetes, al aseguramiento de la cánula mediante ligaduras y a la conexión de ésta a la línea arterial de manera habitual (Figura 3).

La circulación extracorpórea (CEC) es mantenida con los flujos y temperaturas habituales.

En caso de necesidad de paro circulatorio para intervenir el cayado, se procede a disecar el origen de la arteria innominada en el arco aórtico, el que se aísla y asegura con una cinta vascular (Figura 4).

Una vez alcanzada la temperatura deseada se disminuyen los flujos de la CEC al mínimo y se procede al pinzamiento individual de la arteria (Figura 5). Esta maniobra permite aislar y perfundir

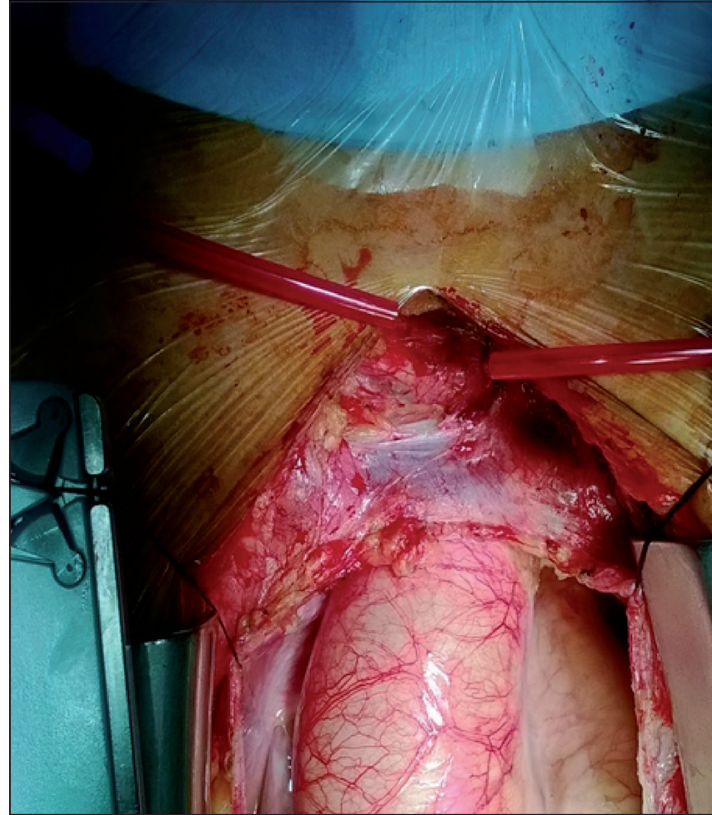

Figura 2. Confección de jaretas.

en forma selectiva la arteria innominada, y a través de ella, las arterias subclavia y carótida derechas. Luego del pinzamiento se restablece la circulación cerebral selectiva a flujos bajos $(600-800 \mathrm{ml} / \mathrm{min})$ regulados según la presión arterial que se mide en la extremidad superior derecha. Cuando ha finalizado el período de paro, se procede a la purga de aire de la aorta y al despinzamiento de la innominada, restableciendo el flujo sistémico.

Una vez terminada la intervención se procede a la desconexión del paciente de la CEC y la decanulación. La entrada de la cánula se cierra simplemente anudando las jaretas (Figura 5).

\section{Comentario}

La canulación de la arteria innominada es una técnica que se puede realizar sin necesidad de una segunda incisión, pues el vaso se expone en la misma esternotomía. La arteria es muy fácil de disecar y habitualmente de gran calibre. Permite entregar un flujo cerebral y visceral anterógrado. En caso de trabajar en el cayado, el pinzamiento de la arteria a nivel de su origen permite entregar perfusión cerebral anterógrada.

Iniciamos esta técnica en el año 2010 en nuestro centro. La hemos realizado tanto en pacientes con cirugía electiva de la aorta ascendente y arco como 


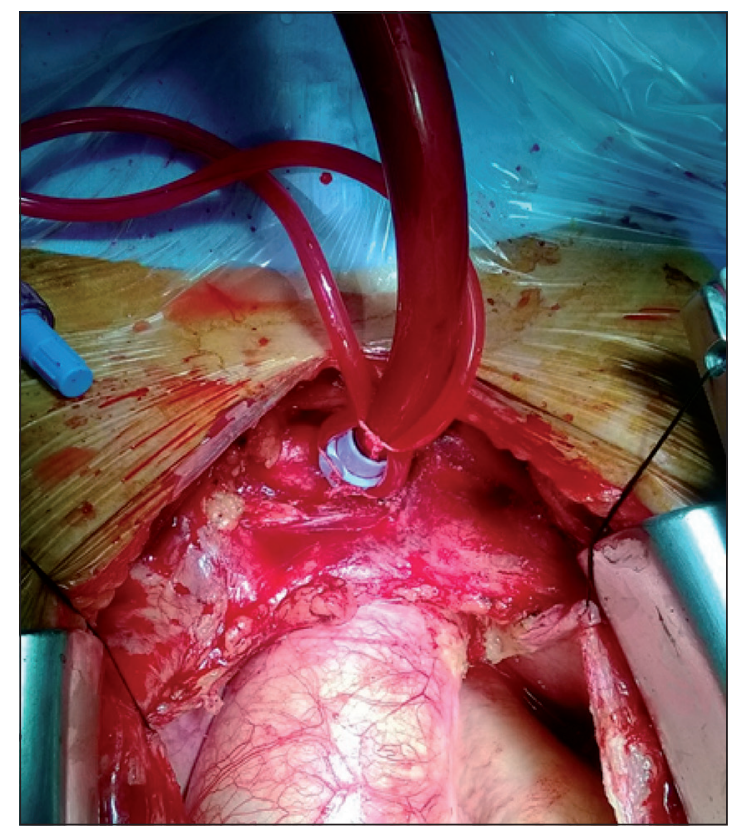

Figura 3. Arteria canulada.

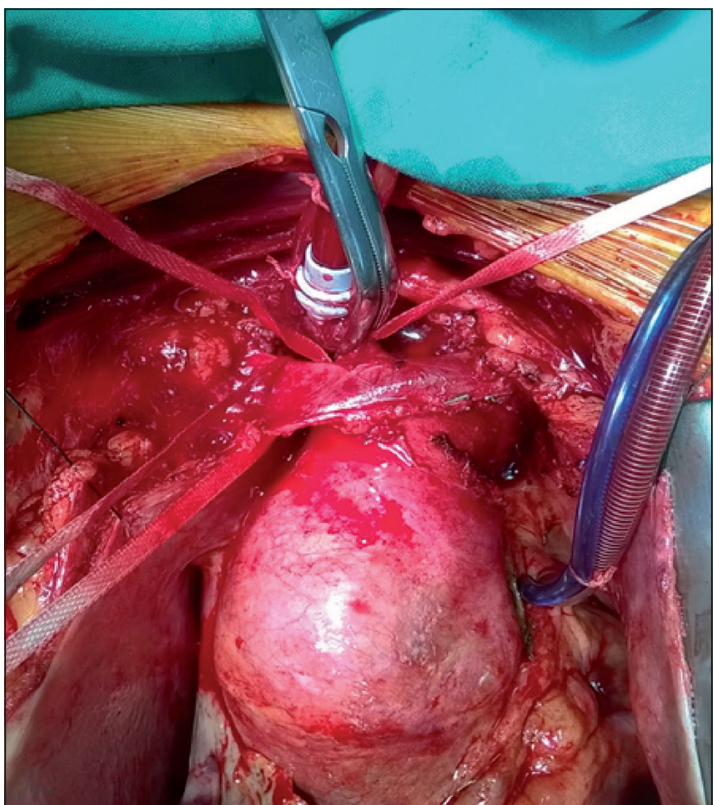

Figura 5. Pinzamiento selectivo de la arteria innominada.

en pacientes con una disección aórtica aguda tipo A. Excepcionalmente la hemos utilizado como alternativa en la canulación en pacientes portadores de enfermedad coronaria y/o valvular que presentan

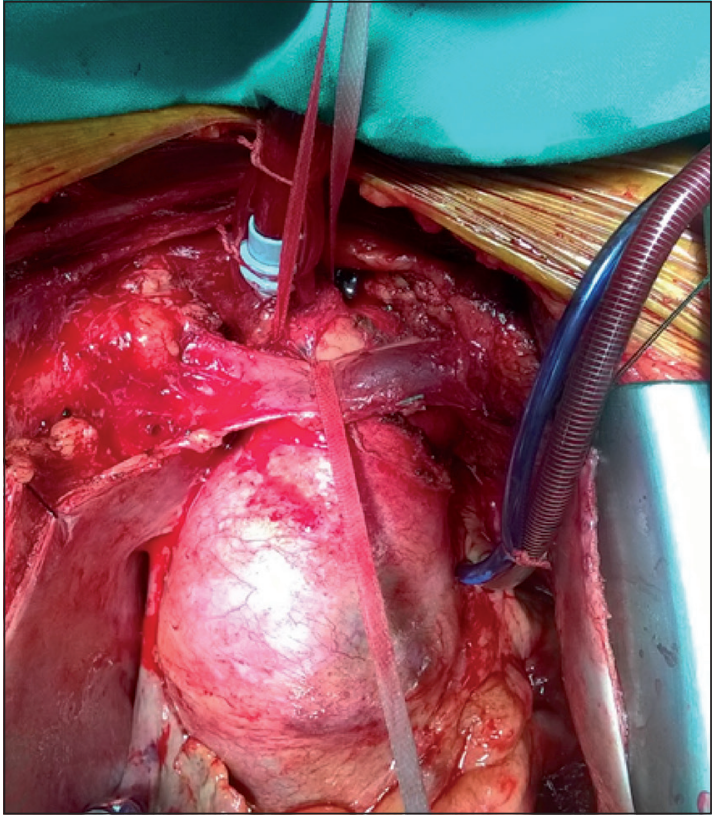

Figura 4. Arteria innominada aislada y controlada con cintas de seda.

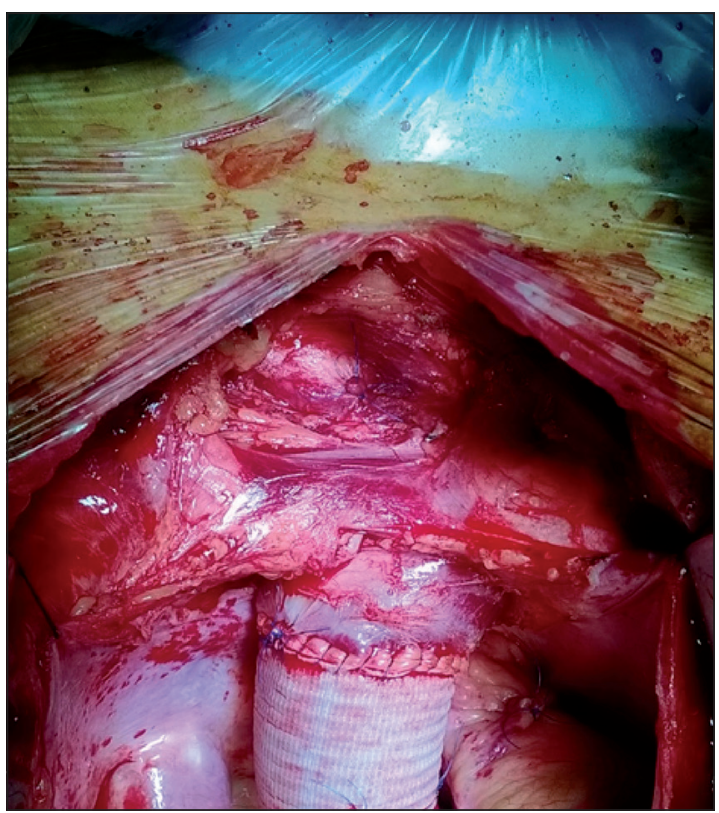

Figura 6. Resultado final posterior al retiro de cánula y cierre de jaretas.

ateromatosis y/o calcificación severa de la aorta ascendente y arco.

Es una técnica fácil y rápida de realizar, y hasta ahora no hemos tenido complicaciones con ella. 
Creemos que es una excelente alternativa de canulación para circulación extra corpórea para la cirugía de la aorta torácica.

\section{Referencias}

1. Dias R, Silva I, Fiorelli A, Stolf A. Cerebral protection: sites of arterial cannulation and brain perfusion routes. Rev Bras Cir Cardiovasc. 2007;22:235-40.

2. Fusco D, Shaw R, Tranquilli M, Kopf G, Elefteriades J. Femoral cannulation is safe for type A dissection repair. Ann Thorac Surg. 2004; 78:1285-9.

3. Bachet J, Guilmet D. Brain protection during surgery of the aortic arch. J Card Surg. 2002;17:115-24.

4. Tiwari K, Murzi M, Bevilacqua S, Glauber M. Which cannulation (ascending aortic cannulation or peripheral arterial cannulation) is better for acute type A aortic dissection surgery? Interact Cardiovasc Thorac Surg. 2010;10:797-802.

5. Kamiya H, Kallenbach K, Halmer D, Ozsöz M, Ilg K, Lichtenberg A, et al. Comparison of ascending aorta versus femoral artery cannulation for acute aortic dissection type A. Circulation 2009;120:S282-S286.

6.- Urbanski P, Lenos A, Lindemann Y, Weigang E, Zacher M, Diegeler A. Carotid artery cannulation in aortic surgery. J Thorac Cardiovasc Surg. 2006;132:1398-403.

7. Mohammadi S, Bonnet N, Leprince P, Kolsi M, Rama A, Pavie A, et al. Reoperation for false aneurysm of the ascending aorta after its prosthetic replacement: surgical strategy. Ann Thorac Surg. 2005;79:147-52.

8. Sabik J, Lytle B, McCarthy P, Cosgrove D. Axillary artery: An alternative site of arterial cannulation for patients with extensive aortic and peripheral vascular disease. J Thorac Cardiovasc Surg. 1995;109:885-90.

9. Reuthebuch O, Schurr U, Hellermann J, Prêtre R, Künzli A, Lachat M, et al. Advantages of subclavian artery perfusion for repair of acute type A dissection. Eur J Cardiothorac Surg. 2004;26:592-8.

10. Schachner T, Nagiller J, Zimmer A, Laufer G, Bonatti J. Technical problems and complications of axillary artery cannulation. Eur J Cardiothorac Surg. 2005;27:634-7.

11. Sabik J, Nemeh H, Lytle B, Blackstone E, Gillinov A, Rajeswaran J, et al. Cannulation of the axillary artery with a side graft reduces morbidity. Ann Thorac Surg. 2004;77:1315-20.

12. Banbury M, Cosgrove D. Arterial cannulation of the innominate artery. Ann Thorac Surg. 2000;69:957.

13. Preventza O, Bakaeen F, Stephens E, Trocciola S, de la Cruz K, Coselli J. Innominate artery cannulation: An alternative to femoral or axillary cannulation for arterial inflow in proximal aortic surgery. J Thorac Cardiovasc Surg. 2013;145(3 Suppl): S191-S196.

14. Di Eusanio M, Ciano M, Labriola G, Lionetti G, Di Eusanio G. Cannulation of the innominate artery during surgery of the thoracic aorta: our experience in 55 patients. Eur J Cardiothorac Surg. 2007;32:270-3.

15. Chiu K, Li S, Lin T, Chan C, Chu S. Innominate artery cannulation for aortic surgery. Asian Cardiovasc Thorac Ann. 2007;15:348-50.

16. Anastasiadis K, Antonitsis P, Papakonstantinou C. Innominate artery cannulation. MMCTS 2008 (1006): mmcts.2008.003418doi: 10.1510/mmcts.2008.003418. 